Article

\title{
Tandem Three-Component Reactions of Aldehyde, Alkyl Acrylate, and Dialkylmalonate Catalyzed by Ethyl Diphenylphosphine
}

\section{Yeong-Jiunn Jang, Siang-En Syu, Yi-Wun Jhang, Yu-Ting Lee, Chia-Jui Lee, Ko-Wei Chen, Utpal Das and Wenwei Lin *}

Department of Chemistry, National Taiwan Normal University No. 88, Section 4, Tingchow Road, Taipei 11677, Taiwan

* Author to whom correspondence should be addressed; E-Mail: wenweilin@ntnu.edu.tw; Tel.: +886-(0)2-2932-4249.

Received: 19 December 2011; in revised form: 9 February 2012 / Accepted: 17 February 2012 / Published: 2 March 2012

\begin{abstract}
A new highly efficient three-component reaction of alkyl acrylate, aldehyde and dialkyl malonate using ethyl diphenylphosphine as organocatalyst has been described. Various highly functional compounds bearing hydroxyl groups and the ester functions can be easily prepared in moderate to good yields according to our one-step procedure. The reactions are believed to proceed via Morita-Baylis-Hillman reactions of alkyl acrylate and aldehydes, followed by the Michael addition reactions of dialkyl malonates. Our reactions indicated that the intermediate species formed in the phosphine-catalyzed MBH reaction are an effective organic base to catalyze the Michael addition reactions of dialkyl malonates to the preformed MBH adducts.
\end{abstract}

Keywords: multicomponent reaction; Morita-Baylis-Hillman; chemoselectivity; Michael addition; aldehyde

\section{Introduction}

Carbon-carbon bond formation is the most important organic reaction because it plays a fundamental role in making carbon-frameworks of organic compounds for numerous interesting studies concerning reactivity, chemoselectivity, and stereoselectivity [1,2]. Multicomponent reactions have long been recognized to play a key role in the development of synthetic methodologies because of 
their possible generation of an adduct in a single step from three or more reactants usually accompanied by bond-forming efficiency and atom economy [3-9]. Successful application of multicomponent reaction highly relies on the good chemoselectivity in the presence of all the reactants [10-12].

The Baylis-Hillman reaction adduct, resulting from alkyl acrylate and aldehyde is a good Michael acceptor because of the activated ester functionality by the neighboring hydroxy group [13-20]. Successful applications of the Baylis-Hillman adducts for further transformation by Michael additions of nucleophiles had been reported [21-24]. However, the Baylis-Hillman reactions are well known for their slow reaction rates and moderate to high yields, and therefore the whole processes often take several days to complete the following Michael reactions [4]. Further effort to simplify the whole process was undertaken by one-pot sequential Baylis-Hillman and Michael reactions with aldehyde, methyl acrylate, and nitroalkane in the presence of DBU (1.0 equiv.) with 26-62\% yields [21], albeit the one-step three-component reaction failed due to the dominant Michael addition of DBU-deprotonated nitroalkane toward methyl acrylate. Therefore, a strong demand remains for an efficient approach.

In continuation of our efforts to simplify the whole process, we envisaged that instead of using a tertiary amine as an organocatalyst, it should be possible to carry out a phosphine-catalyzed three-component reaction starting from the Baylis-Hillman reaction of aldehyde $\mathbf{1}$ and alkyl acrylate $\mathbf{2}$, which is followed by the Michael addition of dialkylmalonate 3 to the resulting adduct [25-28]. Herein, dialkylmalonates 3 become activated by the action of the intermediate species formed in the phosphine-catalyzed $\mathrm{MBH}$ reaction, because phosphines were much poorer bases than amines though the former are stronger nucleophiles [29]. In particular, phosphines are known catalysts capable of promoting Michael reactions in the absence of added bases [30-34]. However, to the best of our knowledge, there is no report of a successful reaction or related study that utilizes $\mathbf{3}$ as the reacting partner. Therefore, we wish to report a highly efficient three-component reaction of $\mathbf{1}$, 2, and $\mathbf{3}$ catalyzed by ethyl diphenylphosphine (Scheme 1).

Scheme 1. A three-component reaction of aromatic aldehyde 1, alkyl acrylate 2, and functional alkane 3 catalysed by $\mathrm{EtPPh}_{2}$.

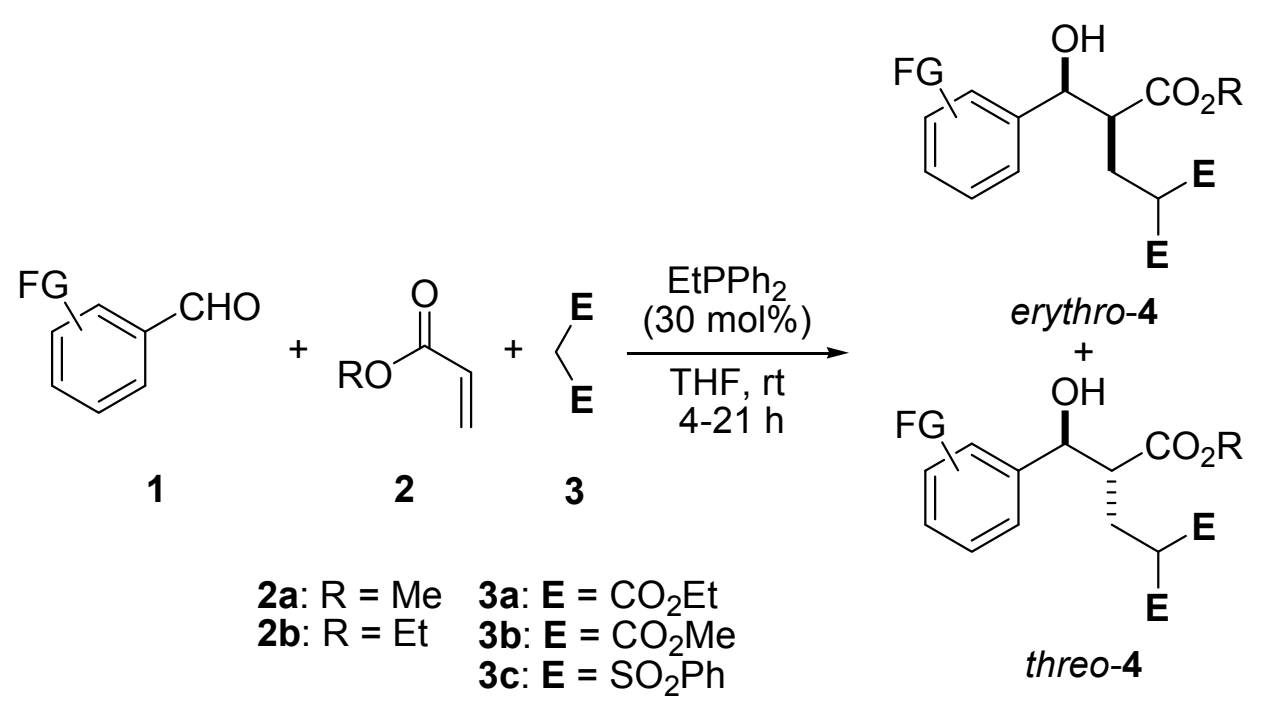




\section{Results and Discussion}

4-Nitrobenzaldehyde (1a), methyl acrylate (2a, 2 equiv.) and diethyl malonate (3a, 1.2 equiv.) were initially chosen and reacted in $t$ - $\mathrm{BuOH}$ in the presence of $\mathrm{EtPPh}_{2}$ at room temperature, providing the densely functionalized three component adduct $\mathbf{4 a}$ in moderate yield (47\%) and good chemoselectivity (21:79 dr) within $0.5 \mathrm{~h}$ (Table 1 , entry 1$)$.

Table 1. Optimization of reaction conditions for a three-component reaction of 4-nitrobenzaldehyde (1a), methyl acrylate (2a) and diethyl malonate (3a) catalyzed by different organocatalysts ${ }^{\text {a }}$.

\begin{tabular}{|c|c|c|c|c|c|c|}
\hline & $2 a$ & $3 a$ & & erythro-4a & \multicolumn{2}{|c|}{ threo-4a } \\
\hline Entry & $\begin{array}{l}\mathrm{CH}_{2}\left(\mathrm{CO}_{2} \mathrm{Et}\right)_{2} \\
\text { (3a) (equiv.) }\end{array}$ & Catalyst & Solvent & Time (h) & dr of $4 a^{b}$ & $\begin{array}{l}\text { Yield of } 4 a \\
(\%)^{c}\end{array}$ \\
\hline 1 & 1.2 & $\mathrm{EtPPh}_{2}$ & $t-\mathrm{BuOH}$ & 0.5 & $21: 79$ & 47 \\
\hline 2 & 1.2 & $\mathrm{PPh}_{3}$ & $t-\mathrm{BuOH}$ & 24 & - & Trace \\
\hline 3 & 1.2 & $\mathrm{PBu}_{3}$ & $t-\mathrm{BuOH}$ & 4 & - & Trace $^{d}$ \\
\hline 4 & 1.2 & DABCO & $t-\mathrm{BuOH}$ & 24 & $33: 67$ & 25 \\
\hline 5 & 1.2 & $\mathrm{EtPPh}_{2}$ & $i-\mathrm{PrOH}$ & 2.5 & $25: 75$ & 38 \\
\hline 6 & 1.2 & $\mathrm{EtPPh}_{2}$ & $\mathrm{CH}_{2} \mathrm{Cl}_{2}$ & 3 & $12: 88$ & 38 \\
\hline 7 & 1.2 & $\mathrm{EtPPh}_{2}$ & Toluene & 4 & $15: 85$ & 51 \\
\hline 8 & 1.2 & $\mathrm{EtPPh}_{2}$ & $\mathrm{THF}$ & 4 & $18: 82$ & 60 \\
\hline 9 & 1.2 & $\mathrm{EtPPh}_{2}{ }^{\mathrm{e}}$ & THF & 2 & $12: 88$ & 49 \\
\hline 10 & 1.2 & $\mathrm{EtPPh}_{2}{ }^{\mathrm{f}}$ & THF & 24 & $21: 79$ & 62 \\
\hline 11 & 1.5 & $\mathrm{EtPPh}_{2}$ & THF & 5 & $16: 84$ & 54 \\
\hline $12^{g}$ & 1.2 & $\mathrm{EtPPh}_{2}$ & $\mathrm{THF} / t-\mathrm{BuOH}^{\mathrm{h}}$ & 1.5 & $19: 81$ & 43 \\
\hline 13 & 1.2 & DMAP & $\mathrm{THF}$ & 24 & - & Trace \\
\hline 14 & 1.2 & DBU & THF & 24 & - & Trace \\
\hline
\end{tabular}

${ }^{\mathrm{a}}$ Unless stated otherwise, the reaction was performed using 4-nitrobenzaldehyde (1a) $(0.5 \mathrm{mmol})$ and methyl acrylate (2a) $(1.0 \mathrm{mmol})$ with $30 \mathrm{~mol} \%$ catalyst in solvent $(0.5 \mathrm{~mL})$ at room temperature; ${ }^{b}$ The diastereometric ratio of $4 \mathbf{a}$ was determined by crude ${ }^{1} \mathrm{H}-\mathrm{NMR}$ analysis; ${ }^{\mathrm{c}}$ Yield of analytically pure isolated product; ${ }^{\mathrm{d}}$ Only significant amount of 4-nitrobenzaldehyde (1a) and diethyl malonate (3a) were observed even after $8 \mathrm{~h}$; ${ }^{\mathrm{e}}$ The reaction was performed with $50 \mathrm{~mol} \%$ $\mathrm{EtPPh}_{2} ;{ }^{\mathrm{f}}$ The reaction was performed with $20 \mathrm{~mol} \% \mathrm{EtPPh}_{2} ;{ }^{\mathrm{g}}$ The reaction was performed at $10{ }^{\circ} \mathrm{C}$; ${ }^{\mathrm{h}} \mathrm{THF} / t-\mathrm{BuOH}=1 / 4$.

Trace amounts of the product 4a were obtained when the less reactive $\mathrm{PPh}_{3}$ was used (entry 2). Significant amounts of 4-nitrobenzaldehyde (1a) and diethyl malonate (3a) were recovered, because the acrylate was prone to undergo polymerization when the extremely active $\mathrm{PBu}_{3}$ was used as the catalyst (entry 3). DABCO, which has weaker nucleophilicity than that of $\mathrm{EtPPh}_{2}$, catalyzed the three component reaction of 1a, 2a and 3a and afforded 4a in only $25 \%$ yield within $24 \mathrm{~h}$ (entry 4). Inferior results were obtained when the reactions were carried out in $i-\mathrm{PrOH}, \mathrm{CH}_{2} \mathrm{Cl}_{2}$ and toluene (entries 5-7). 
Interestingly, when a polar aprotic solvent was used, such as THF, a significantly increased yield was observed (4a, $60 \%$ yield, entry 8). Increasing the amount of $\mathrm{EtPPh}_{2}$, prolonging the reaction time with 1.5 equiv. of $3 \mathbf{a}$, or using a co-solvent system $(\mathrm{THF} / \mathrm{t}-\mathrm{BuOH}=1 / 4)$ did not improve the final results either (entries 9,11 and 12), and furthermore a worse diastereometric excess resulted from decreasing the amount of $\mathrm{EtPPh}_{2}$ (entry 10). DMAP and DBU were also examined, but only trace amounts of adduct 4 a could be observed (entries 13 and 14).

The broad reaction scope of our protocol was demonstrated by further studies disclosed in Table 2 . It showed that chemoselective three-component reactions of various aromatic aldehydes $\mathbf{1 a}-\mathbf{f}$, alkyl acrylate 2 ( 2 equiv.), and $3 \mathbf{a}$ (1.2 equiv.) in the presence of $\mathrm{EtPPh}_{2}(30 \mathrm{~mol} \%)$ took place in 4-21 h, leading to the corresponding adducts $4 \mathbf{a}-\mathbf{j}$ in $36-63 \%$ yields.

Table 2. A three component reaction of aromatic aldehyde 1, alkyl acrylate 2, and dialkyl malonate 3 catalysed by $\mathrm{EtPPh}_{2}$ a

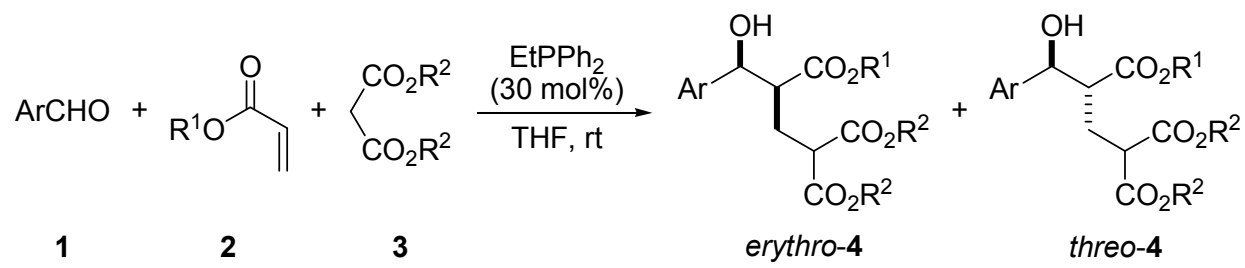

\begin{tabular}{|c|c|c|c|c|c|c|}
\hline Entry & Ar & $\mathbf{R}^{1}$ & $\mathbf{R}^{2}$ & Time (h) & dr of $4^{b}$ & Yield of $4(\%)^{c}$ \\
\hline 1 & $4-\mathrm{NO}_{2} \mathrm{C}_{6} \mathrm{H}_{4}(\mathbf{1 a})$ & $\mathrm{CH}_{3}(\mathbf{2 a})$ & $\mathrm{C}_{2} \mathrm{H}_{5}(\mathbf{3 a})$ & 4 & $18: 82$ & $4 \mathbf{a}(60)$ \\
\hline 2 & $3-\mathrm{NO}_{2} \mathrm{C}_{6} \mathrm{H}_{4}(\mathbf{1 b})$ & $\mathbf{2 a}$ & $\mathbf{3 a}$ & 4 & $13: 87$ & $4 b(63)$ \\
\hline 3 & $2-\mathrm{NO}_{2} \mathrm{C}_{6} \mathrm{H}_{4}(\mathbf{1 c})$ & $2 \mathbf{a}$ & $3 \mathbf{a}$ & 4 & $37: 63$ & $4 c(36)$ \\
\hline 4 & $4-\mathrm{CF}_{3} \mathrm{C}_{6} \mathrm{H}_{4}(\mathbf{1 d})$ & $2 a$ & $3 a$ & 10 & $20: 80$ & $4 d(57)$ \\
\hline 5 & $4-\mathrm{CNC}_{6} \mathrm{H}_{4}(\mathbf{1 e})$ & $2 \mathbf{a}$ & $3 \mathbf{a}$ & 4 & $17: 83$ & $4 \mathbf{e}(50)$ \\
\hline 6 & 3-Pyridyl (1f) & $2 \mathbf{a}$ & $3 a$ & 21 & $18: 82$ & $\mathbf{4 f}(55)$ \\
\hline 7 & $4-\mathrm{NO}_{2} \mathrm{C}_{6} \mathrm{H}_{4}(\mathbf{1 a})$ & $\mathrm{C}_{2} \mathrm{H}_{5}(\mathbf{2 b})$ & $3 \mathbf{a}$ & 4 & $9: 91$ & $4 \mathbf{g}(50)$ \\
\hline 8 & $3-\mathrm{NO}_{2} \mathrm{C}_{6} \mathrm{H}_{4}(\mathbf{1 b})$ & $2 \mathbf{b}$ & $3 \mathbf{a}$ & 4 & $15: 85$ & $4 h(53)$ \\
\hline 9 & $4-\mathrm{CNC}_{6} \mathrm{H}_{4}(\mathbf{1 e})$ & $2 \mathbf{b}$ & $3 \mathbf{a}$ & 6 & $11: 89$ & $4 \mathbf{i}(51)$ \\
\hline 10 & 3-Pyridyl (1f) & $2 \mathbf{b}$ & $\mathbf{3 a}$ & 20 & $14: 86$ & $4 \mathbf{j}(52)$ \\
\hline 11 & $4-\mathrm{NO}_{2} \mathrm{C}_{6} \mathrm{H}_{4}(\mathbf{1 a})$ & $\mathrm{CH}_{3}(\mathbf{2 a})$ & $\mathrm{CH}_{3}(\mathbf{3 b})$ & 5 & $13: 87$ & 4k (39) \\
\hline
\end{tabular}

${ }^{\mathrm{a}}$ Unless stated otherwise, the reaction was performed using $\mathbf{1}(0.5 \mathrm{mmol}), \mathbf{2}$ (2.0 equiv.), and 3a (1.2 equiv.) in the presence of $\mathrm{EtPPh}_{2}(30 \mathrm{~mol} \%)$ in THF $(0.5 \mathrm{~mL})$ at room temperature; ${ }^{\mathrm{b}}$ The diastereomeric ratio of $\mathbf{4}$ was determined by ${ }^{1} \mathrm{H}-\mathrm{NMR}$ analysis. The stereochemistry of $\mathbf{4 a}-\mathbf{b}, \mathbf{4 d}$, $\mathbf{4 e}$, and $\mathbf{4 g - i}$ was determined by ${ }^{1} \mathrm{H}-\mathrm{NMR}$ analysis in comparison to $\mathbf{5}$. For $\mathbf{4 c}, \mathbf{4 f}$, and $\mathbf{4 j}$, their stereochemistry is not determined; ${ }^{\mathrm{c}}$ Yield of analytically pure isolated product.

A steric effect was found in case of reactions with an ortho-substituted aromatic aldehydes. For example, aromatic aldehydes bearing a nitro group in the para- or meta-position, like 1a or $\mathbf{1 b}$, reacted with $\mathbf{2 a}$ and 3a within $4 \mathrm{~h}$ to provide the corresponding adducts $\mathbf{4 a}$ or $\mathbf{4 b}$ with good stereoselectivities $(18: 82 \mathrm{dr}, 13: 87 \mathrm{dr}$ ) in $60 \%$ or $63 \%$ yield, respectively (entries 1 and 2). However, in the case of 2-nitrobenzaldehyde (1c), poor results for the formation of $4 \mathbf{c}$ were obtained (36\% yield and 37:63 dr, entry 3). Other substituted aromatic aldehydes, such as 4-trifluoromethylbenzaldehyde (1d) or 4-cyanobenzaldehyde (1e), reacted like $\mathbf{2 a}$ and $\mathbf{3 a}$, generating $\mathbf{4 d}$ or $\mathbf{4 e}$ in $57 \%$ or $50 \%$ yield with 20:80 dr or 17:83 dr, resepectively (entries 4 and 5). Moderate yield and good stereoselectivity of $\mathbf{4 f}$ 
was also produced when heteroaromatic aldehyde 1f was employed in our protocol (entry 6). The reaction of 1a, 1b, 1e, or 1f and 3a also proceeded well when ethyl acrylate (2b) was used as the activated olefin, furnishing the corresponding adducts $4 \mathbf{g}, \mathbf{4 h}, \mathbf{4 i}$, or $4 \mathbf{j}$ in moderate yield with high stereoselectivity (entries 7-10). In addition to diethyl malonate (3a), dimethyl malonate (3b) and bis(phenylsulfonyl)methane (3c) were also investigated as carbon nucleophiles for this protocol. Both of them worked smoothly with 4-nitrobenzaldehyde (1a) and methyl acrylate (2a, 2 equiv.) in the presence of $\mathrm{EtPPh}_{2}$ to give the corresponding adducts $4 \mathbf{k}$ (39\% yield, 13:87 dr) and $\mathbf{5}$ (54\% yield, 47:53 dr) (entry 11 and Scheme 2) [35].

Scheme 2. A three-component reaction of 4-nitrobenzaldehyde (1a), methyl acrylate (2a, 2 equiv.) and bis(phenylsulfonyl)methane (3c, 1.2 equiv.) catalyzed by $\mathrm{EtPPh}_{2}$.

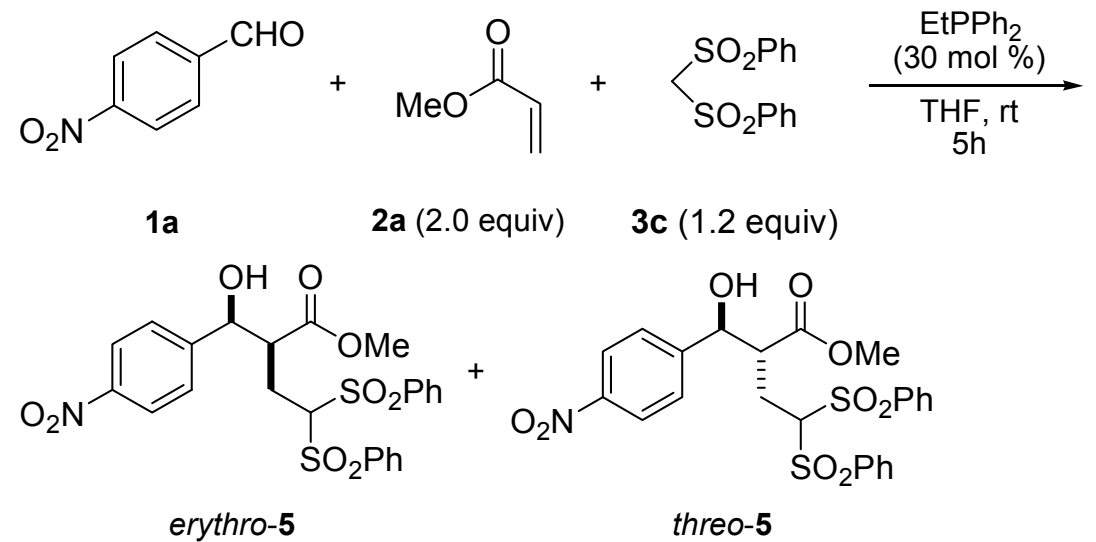

$54 \%$ yield $(\mathrm{dr}=47: 53)$

Remarkably, a tandem three-component reaction of 4-nitrobenzaldehyde (1a), methyl acrylate (2a, 1.2 equiv.) and a sterically bulky carbon nucleophile, diethyl methylmalonate (3d) (1.7 equiv.), ocurred successfully at room temperature within $9 \mathrm{~h}$ to provide the desired product $\mathbf{6 n}$ in $57 \%$ yield with good stereoselectivity (Scheme 3).

Scheme 3. A three-component reaction of 4-nitrobenzaldehyde (1a), methyl acrylate (2a, 1.2 equiv.), and diethyl methylmalonate (3d, 1.7 equiv.) catalyzed by $\mathrm{EtPPh}_{2}$.

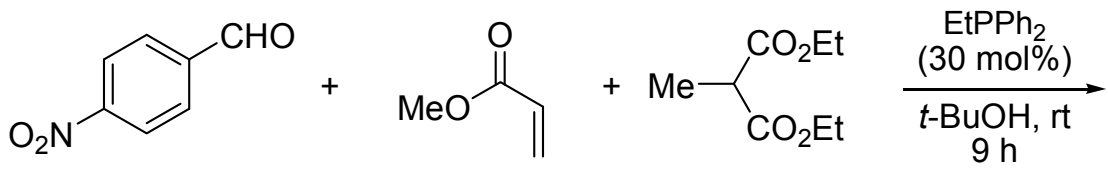

$1 \mathrm{a}$

2a (1.2 equiv) $\quad 3 d(1.7$ equiv)<smiles>CCOC(C)C(CC(C)(OCC)OCC)C(O)c1ccc([N+](=O)[O-])cc1</smiles>

erythro-6<smiles>CCOC(C)(C)C[C@H](C(C)=O)C(O)c1ccc([N+](=O)[O-])cc1</smiles>

threo-6 


\section{Experimental}

\subsection{General}

All reactions were carried out under a nitrogen atmosphere in dried glassware. All starting materials were purchased from commercial sources and used without further purification. THF was continuously refluxed and freshly distilled from sodium benzophenone ketyl under nitrogen. $t$-BuOH was dried and degassed before use. Yields refer to isolated yields of compounds estimated to be $>95 \%$ pure as determined by ${ }^{1} \mathrm{H}-\mathrm{NMR}$ in a AV-400 or AV-500 Bruker using $\mathrm{CDCl}_{3}$ as solvent at $400 \mathrm{~Hz}$, respectively. MS and HRMS were recorded in a Finnigan TSQ 700 and JEOL JMS-700 mass spectrometers. Analytical thin layer chromatography (TLC) was performed using Merck 60 F254 precoated silica gel plate $(0.2 \mathrm{~mm}$ thickness). Flash chromatography was performed using Merck silica gel 60 .

\subsection{Typical Synthetic Procedure}

3.2.1. Optimization of Reaction Conditions for an Organocatalytic Three-Component Reaction of 4-Nitrobenzaldehyde (1a), Methyl Acrylate (2a), and Diethyl Malonate (3a) (TP for Table 1)

A dry and nitrogen-flushed 10-mL Schlenk flask, equipped with a magnetic stirring bar and a septum, was charged with a solution of $\mathbf{1 a}(75.6 \mathrm{mg}, 0.5 \mathrm{mmol})$ and $\mathbf{3 a}(91.0 \mu \mathrm{L}, 1.2$ equiv.) in solvent (dry degassed $t$-BuOH, $i$-PrOH or dry THF) $(0.5 \mathrm{~mL})$. Methyl acrylate (2a) $(90.0 \mu \mathrm{L}, 2.0$ equiv.) and catalyst $\left(\mathrm{EtPPh}_{2}, \mathrm{PPh}_{3}, \mathrm{PBu}_{3}, \mathrm{DABCO}, \mathrm{DMAP}\right.$ or $\left.\mathrm{DBU}\right)(30 \mathrm{~mol} \%)$ were added, and the reaction mixture was stirred for indicated time at room temperature. Thereafter, the solvent was removed by evaporation in vacuo. Purification by flash chromatography ( $n$-hexanes/ethyl acetate $4: 1$ ) furnished the adduct $4 \mathbf{a}$.

3.2.2. Typical Procedure for a Three-Component Reaction of Aromatic Aldehyde, Alkyl Acrylate, and Diethyl Malonate Catalyzed by $\mathrm{EtPPh}_{2}$ (TP for Table 2)

A dry and nitrogen-flushed 10-mL Schlenk flask, equipped with a magnetic stirring bar and a septum, was charged with a solution of $1(0.5 \mathrm{mmol})$ and $\mathbf{3 a}$ or $\mathbf{3 b}$ (1.2 equiv.) in dry degassed THF (0.5 mL). Alkyl acrylate $2 \mathrm{a}$ or $2 \mathrm{~b}\left(2.0\right.$ equiv.) and $\mathrm{EtPPh}_{2}(30.7 \mu \mathrm{L}, 30 \mathrm{~mol} \%)$ were added, and the reaction mixture was stirred for 4-21 h at room temperature. Thereafter, the solvent was removed by evaporation in vacuo. Purification by flash chromatography furnished the adducts erythro-4 and threo-4.

\subsubsection{Procedure for Preparation of 5 (TP for Scheme 2)}

A dry and nitrogen-flushed 10-mL Schlenk flask, equipped with a magnetic stirring bar and a septum, was charged with a solution of $\mathbf{1 a}(151.1 \mathrm{mg}, 1.0 \mathrm{mmol})$ and $\mathbf{3 c}(355.6 \mathrm{mg}, 1.2 \mathrm{equiv}$.) in dry THF (1.0 mL). Methyl acrylate $2 \mathrm{a}(180.0 \mu \mathrm{L}, 2.0$ equiv. $)$ and $\mathrm{EtPPh}_{2}(61.0 \mu \mathrm{L}, 30 \mathrm{~mol} \%)$ were added, and the reaction mixture was stirred for $5 \mathrm{~h}$ at room temperature. Thereafter, the solvent was removed by evaporation in vacuo. Purification by flash chromatography furnished the adducts erythro-5 and threo-5 (dr = 47:53, $288.1 \mathrm{mg}, 54 \%)$. 


\subsubsection{Procedure for Preparation of 6 (TP for Scheme 3)}

A dry and nitrogen-flushed 10-mL Schlenk flask, equipped with a magnetic stirring bar and a septum, was charged with a solution of $\mathbf{1 a}(0.5 \mathrm{mmol})$ and $\mathbf{3 d}(102.3 \mu \mathrm{L}, 1.2$ equiv. $)$ in dry $t$-BuOH $(0.5 \mathrm{~mL})$. Methyl acrylate $2 \mathbf{a}\left(90.0 \mu \mathrm{L}, 2.0\right.$ equiv.) and $\mathrm{EtPPh}_{2}(30.7 \mu \mathrm{L}, 30 \mathrm{~mol} \%)$ were added, and the reaction mixture was stirred for $9 \mathrm{~h}$ at room temperature. Thereafter, the solvent was removed by evaporation in vacuo. Purification by flash chromatography furnished the adducts $6(\mathrm{dr}=79: 21,117.1 \mathrm{mg}, 57 \%)$.

Erythro-1,1-diethyl 3-methyl 4-hydroxy-4-(4-nitrophenyl)butane-1,1,3-tricarboxylate (erythro-4a). ${ }^{1} \mathrm{H}-\mathrm{NMR}\left(400 \mathrm{MHz}, \mathrm{CDCl}_{3}, 25{ }^{\circ} \mathrm{C}\right) \delta / \mathrm{ppm}: 8.18(\mathrm{~d}, 2 \mathrm{H}, J=8.4 \mathrm{~Hz}), 7.52(\mathrm{~d}, 2 \mathrm{H}, J=8.4 \mathrm{~Hz}), 5.13(\mathrm{~d}$, $1 \mathrm{H}, J=3.7 \mathrm{~Hz}), 4.22-4.01(\mathrm{~m}, 4 \mathrm{H}), 3.63(\mathrm{~s}, 3 \mathrm{H}), 3.44(\mathrm{~s}, 1 \mathrm{H}), 3.40-3.27(\mathrm{~m}, 1 \mathrm{H}), 2.89-2.76(\mathrm{~m}, 1 \mathrm{H})$, 2.36-2.20 (m, 1H), 2.17-2.01 (m, 1H), 1.27-1.08 (m, 6H); ${ }^{13} \mathrm{C}-\mathrm{NMR}\left(100 \mathrm{MHz}, \mathrm{CDCl}_{3}, 25{ }^{\circ} \mathrm{C}\right)$

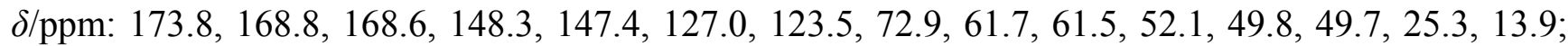
MS (70 eV, EI) m/z (\%): 398 (2) [M+1] $]^{+}, 380$ (8), 319 (80), 302 (77), 274 (43), 245 (72), 160 (100), 133 (82), 127 (85), 113 (48), 105 (52), 55 (81); HRMS (MALDI) for $\mathrm{C}_{18} \mathrm{H}_{23} \mathrm{NO}_{9} \mathrm{Na},[\mathrm{M}+\mathrm{Na}]^{+}$ (420.1270) found: 420.1275.

Threo-1,1-diethyl 3-methyl 4-hydroxy-4-(4-nitrophenyl)butane-1,1,3-tricarboxylate (threo-4a). ${ }^{1} \mathrm{H}-\mathrm{NMR}\left(400 \mathrm{MHz}, \mathrm{CDCl}_{3}, 25{ }^{\circ} \mathrm{C}\right) \delta / \mathrm{ppm}: 8.22(\mathrm{~d}, 2 \mathrm{H}, J=8.8 \mathrm{~Hz}), 7.50(\mathrm{~d}, 2 \mathrm{H}, J=8.6 \mathrm{~Hz}), 4.98(\mathrm{~d}$, $1 \mathrm{H}, J=5.8 \mathrm{~Hz}), 4.23-4.14(\mathrm{~m}, 4 \mathrm{H}), 3.64(\mathrm{~s}, 3 \mathrm{H}), 3.38(\mathrm{dd}, 1 \mathrm{H} J=5.7 \mathrm{~Hz}, 9.3 \mathrm{~Hz}), 2.97-2.90(\mathrm{~m}, 1 \mathrm{H})$, 2.30-2.21 (m, 1H), 2.18-2.09 (m, 1H), 1.29-1.21 (m, 6H); ${ }^{13} \mathrm{C}-\mathrm{NMR}\left(100 \mathrm{MHz}, \mathrm{CDCl}_{3}, 25{ }^{\circ} \mathrm{C}\right)$ $\delta /$ ppm: 173.8, 168.6, 168.5, 148.7, 147.6, 127.1, 123.7, 73.8, 61.8, 61.7, 52.1, 49.8, 49.6, 27.9, 14.0; MS (70 eV, EI) m/z (\%): 398 (1) [M+1], 380 (5), 319 (85), 302 (79), 274 (40), 245 (77), 223 (86), 160 (100), 133 (93), 127 (85), 113 (51), 105 (49), 55 (81); HRMS (MALDI) for $\mathrm{C}_{18} \mathrm{H}_{23} \mathrm{NO}_{9} \mathrm{Na},[\mathrm{M}+\mathrm{Na}]^{+}$ (420.1270) found: 420.1283.

Erythro-1,1-diethyl 3-methyl 4-hydroxy-4-(3-nitrophenyl)butane-1,1,3-tricarboxylate (erythro-4b). ${ }^{1} \mathrm{H}-\mathrm{NMR}\left(400 \mathrm{MHz}, \mathrm{CDCl}_{3}, 25{ }^{\circ} \mathrm{C}\right) \delta / \mathrm{ppm}: 8.23(\mathrm{~s}, 1 \mathrm{H}), 8.17-8.11(\mathrm{~m}, 1 \mathrm{H}), 7.69(\mathrm{~d}, 1 \mathrm{H}, J=7.7 \mathrm{~Hz})$, 7.52 (t, $1 \mathrm{H} J=7.9 \mathrm{~Hz}$ ), 5.14 (pseudo t, $1 \mathrm{H}, J=4.1 \mathrm{~Hz}), 4.22-4.06(\mathrm{~m}, 4 \mathrm{H}), 3.65$ (s, 3H), 3.37 (dd, $1 \mathrm{H}$ $J=9.3 \mathrm{~Hz}, 5.3 \mathrm{~Hz}), 2.90-2.82(\mathrm{~m}, 1 \mathrm{H}), 2.35-2.25$ (m, 1H), 2.18-2.07 (m, 1H), 1.28-1.14 (m, 6H).

${ }^{13} \mathrm{C}-\mathrm{NMR}\left(100 \mathrm{MHz}, \mathrm{CDCl}_{3}, 25{ }^{\circ} \mathrm{C}\right) \delta / \mathrm{ppm}: 173.9,168.9,168.7,148.3,143.2,132.2,129.3,122.8$, 121.2, 72.7, 61.7, 61.6, 52.2, 49.9, 49.8, 25.4, 13.9. MS (20 eV, EI) $m / z(\%): 398(5)[\mathrm{M}+1]^{+}, 380$ (10), 348 (5), 246 (41), 214 (11), 187 (11), 173 (7), 160 (100), 134 (20). HRMS (ESI) for $\mathrm{C}_{18} \mathrm{H}_{23} \mathrm{NO}_{9} \mathrm{Na}$, $[\mathrm{M}+\mathrm{Na}]^{+}(420.1270)$ found: 420.1268 .

Threo-1,1-diethyl 3-methyl 4-hydroxy-4-(3-nitrophenyl)butane-1,1,3-tricarboxylate (threo-4b). ${ }^{1} \mathrm{H}-\mathrm{NMR}\left(400 \mathrm{MHz}, \mathrm{CDCl}_{3}, 25{ }^{\circ} \mathrm{C}\right) \delta / \mathrm{ppm}: 8.16(\mathrm{~s}, 1 \mathrm{H}), 8.15-8.08(\mathrm{~m}, 1 \mathrm{H}), 7.65(\mathrm{~d}, 1 \mathrm{H}, J=7.7 \mathrm{~Hz})$, 7.51 (t, $1 \mathrm{H} J=7.9 \mathrm{~Hz}$ ), 4.95 (pseudo s, 1H), 4.18-4.07 (m, 4H), 3.63 (s, 3H), 3.60 (brs, 1H), 3.33 (dd, $1 \mathrm{H} J=9.4 \mathrm{~Hz}, 5.6 \mathrm{~Hz}), 2.95-2.85(\mathrm{~m}, 1 \mathrm{H}), 2.24-2.12(\mathrm{~m}, 1 \mathrm{H}), 2.08-1.98(\mathrm{~m}, 1 \mathrm{H}), 1.25-1.15(\mathrm{~m}, 6 \mathrm{H})$. ${ }^{13} \mathrm{C}-\mathrm{NMR}\left(100 \mathrm{MHz}, \mathrm{CDCl}_{3}, 25{ }^{\circ} \mathrm{C}\right) \delta / \mathrm{ppm}: 173.7,168.6,168.4,148.2,143.6,132.3,129.4,122.9$, $121.2,73.7,61.7,61.6,52.0,50.0,49.6,27.7,13.9,13.7 . \mathrm{MS}(20 \mathrm{eV}, \mathrm{EI}) \mathrm{m} / z(\%): 398(1)[\mathrm{M}+1]^{+}, 380$ (5), 348 (5), 301 (6), 246 (41), 214 (8), 187 (11), 173 (8), 160 (100), 134 (5). HRMS (ESI) for $\mathrm{C}_{18} \mathrm{H}_{23} \mathrm{NO}_{9} \mathrm{Na}$, [M+Na] ${ }^{+}$(420.1270) found: 420.1273 . 
Erythro-1,1-diethyl 3-methyl 4-hydroxy-4-(2-nitrophenyl)butane-1,1,3-tricarboxylat (erythro-4c). ${ }^{1} \mathrm{H}-\mathrm{NMR}\left(400 \mathrm{MHz}, \mathrm{CDCl}_{3}, 25{ }^{\circ} \mathrm{C}\right) \delta / \mathrm{ppm}: 8.00(\mathrm{~d}, 1 \mathrm{H}, J=8.2 \mathrm{~Hz}), 7.89(\mathrm{~d}, 1 \mathrm{H}, J=7.8 \mathrm{~Hz}), 7.68$ (t, $1 \mathrm{H} J=7.6 \mathrm{~Hz}$ ), 7.47 (t, 1H $J=7.8 \mathrm{~Hz}$ ), 5.72 (pseudo s, 1H), 4.19-4.10 (m, 2H), 4.07 (quart, $2 \mathrm{H}$ $J=7.1 \mathrm{~Hz}), 3.69(\mathrm{~s}, 3 \mathrm{H}), 3.43(\mathrm{~d}, 1 \mathrm{H}, J=3.0 \mathrm{~Hz}), 3.36(\mathrm{dd}, 1 \mathrm{H}, J=9.8 \mathrm{~Hz}, J=5.3 \mathrm{~Hz}), 2.98(\mathrm{dt}, 1 \mathrm{H}$, $J=10.4 \mathrm{~Hz}, J=3.8 \mathrm{~Hz}), 2.38-2.28(\mathrm{~m}, 1 \mathrm{H}), 2.17-2.08(\mathrm{~m}, 1 \mathrm{H}), 1.23(\mathrm{t}, 3 \mathrm{H}, J=7.2 \mathrm{~Hz}), 1.15(\mathrm{t}, 3 \mathrm{H}$, $J=7.1 \mathrm{~Hz}) .{ }^{13} \mathrm{C}-\mathrm{NMR}\left(100 \mathrm{MHz}, \mathrm{CDCl}_{3}, 25{ }^{\circ} \mathrm{C}\right) \delta / \mathrm{ppm}: 174.6,168.9,168.6,147.5,136.1,133.5$, 129.3, 128.7, 124.9, 69.2, 61.6, 61.5, 52.2, 49.9, 47.6, 25.1, 13.9. MS (20 eV, EI) m/z (\%): 398 (100) $[\mathrm{M}+1]^{+}, 385$ (62), 380 (61), 363 (75). HRMS (FAB) for $\mathrm{C}_{18} \mathrm{H}_{24} \mathrm{NO}_{9},[\mathrm{M}+\mathrm{H}]^{+}$(398.1451) found: 398.1457 .

Threo-1,1-diethyl 3-methyl 4-hydroxy-4-(2-nitrophenyl)butane-1,1,3-tricarboxylate (threo-4c). ${ }^{1} \mathrm{H}-\mathrm{NMR}\left(400 \mathrm{MHz}, \mathrm{CDCl}_{3}, 2{ }^{\circ} \mathrm{C}\right) \delta / \mathrm{ppm}: 7.98(\mathrm{dd}, 1 \mathrm{H}, J=8.0 \mathrm{~Hz}, J=0.6 \mathrm{~Hz}), 7.71-7.61(\mathrm{~m}, 2 \mathrm{H})$, $7.49-7.42(\mathrm{~m}, 1 \mathrm{H}), 5.46(\mathrm{dd}, 1 \mathrm{H}, J=6.7 \mathrm{~Hz}, J=5.2 \mathrm{~Hz}), 4.25-4.14(\mathrm{~m}, 4 \mathrm{H}), 3.84(\mathrm{~d}, 1 \mathrm{H} J=7.5 \mathrm{~Hz}), 3.58$ (s, 3H), $3.43(\mathrm{dd}, 1 \mathrm{H}, J=8.9 \mathrm{~Hz}, J=6.2 \mathrm{~Hz}), 3.11-3.04(\mathrm{~m}, 1 \mathrm{H}), 2.45-2.34(\mathrm{~m}, 1 \mathrm{H}), 2.30-2.20$ (m, 1H), $1.30-1.22(\mathrm{~m}, 6 \mathrm{H}) .{ }^{13} \mathrm{C}-\mathrm{NMR}\left(100 \mathrm{MHz}, \mathrm{CDCl}_{3}, 25{ }^{\circ} \mathrm{C}\right) \delta / \mathrm{ppm}: 174.3,168.9,168.8,148.1$, 137.4, 133.7, 129.0, 128.6, 125.1, 70.2, 61.9, 52.1, 49.9, 48.9, 28.8, 14.1. MS (FAB) $m / z(\%): 398$ (100) $[\mathrm{M}+1]^{+}, 385$ (60), 380 (65), 363 (72). HRMS (FAB) for $\mathrm{C}_{18} \mathrm{H}_{24} \mathrm{NO}_{9},[\mathrm{M}+\mathrm{H}]^{+}$(398.1451) found: 398.1454 .

Erythro-1,1-diethyl 3-methyl 4-hydroxy-4-(4-(trifluoromethyl)phenyl)butane-1,1,3-tricarboxylate (erythro-4d). ${ }^{1} \mathrm{H}-\mathrm{NMR}\left(400 \mathrm{MHz}, \mathrm{CDCl}_{3}, 25^{\circ} \mathrm{C}\right) \delta / \mathrm{ppm}: 7.60$ (d, $\left.2 \mathrm{H}, J=8.2 \mathrm{~Hz}\right), 7.47$ (d, 2H, $\left.J=8.1 \mathrm{~Hz}\right)$, 5.10 (pseudo t, $1 \mathrm{H}, J=3.9 \mathrm{~Hz}), 4.19-4.03(\mathrm{~m}, 4 \mathrm{H}), 3.64(\mathrm{~s}, 3 \mathrm{H}), 3.36$ (dd, 1H, $J=5.4 \mathrm{~Hz}, 9.5 \mathrm{~Hz}$ ), $3.17(\mathrm{~d}, J=3.4 \mathrm{~Hz}), 2.88-2.79(\mathrm{~m}, 1 \mathrm{H}), 2.35-2.24(\mathrm{~m}, 1 \mathrm{H}), 2.19-2.09(\mathrm{~m}, 1 \mathrm{H}), 1.22(\mathrm{t}, 3 \mathrm{H}, J=7.2 \mathrm{~Hz})$, $1.17(\mathrm{t}, 3 \mathrm{H}, J=7.2 \mathrm{~Hz}) .{ }^{13} \mathrm{C}-\mathrm{NMR}\left(100 \mathrm{MHz}, \mathrm{CDCl}_{3}, 25{ }^{\circ} \mathrm{C}\right) . \delta / \mathrm{ppm}: 174.1,168.9,168.7,144.9,130.0$ (quartet, $J=32 \mathrm{~Hz}$ ), 126.7, 125.2 (quartet, $J=4 \mathrm{~Hz}$ ), 123.9 (quartet, $J=270 \mathrm{~Hz}$ ), 130.0 (quartet, $J=33 \mathrm{~Hz}$ ), 126.4, 125.3 (quartet, $J=3 \mathrm{~Hz}$ ), 123.8 (quartet, $J=269 \mathrm{~Hz}$ ), 73.1, 61.6, 61.5, 52.1, 49.9, 49.8, 25.4, 14.0, 13.9. MS (FAB) $m / z$ (\%): 420 (1) [M] $]^{+}, 403$ (10), 360 (58), 245 (42), 173 (31), 160 (100), 126 (38), 55 (20). HRMS (ESI) for $\mathrm{C}_{19} \mathrm{H}_{23} \mathrm{~F}_{3} \mathrm{NaO}_{7}$, [M+Na] $]^{+}$(443.1294) found: 400.1290.

Threo-1,1-diethyl 3-methyl 4-hydroxy-4-(4-(trifluoromethyl)phenyl)butane-1,1,3-tricarboxylate (threo4d). ${ }^{1} \mathrm{H}-\mathrm{NMR}\left(400 \mathrm{MHz}, \mathrm{CDCl}_{3}, 25{ }^{\circ} \mathrm{C}\right) \delta / \mathrm{ppm}: 7.57$ (d, 2H, $\left.J=8.2 \mathrm{~Hz}\right), 7.40(\mathrm{~d}, 2 \mathrm{H}, J=8.1 \mathrm{~Hz})$, $4.85(\mathrm{~d}, 1 \mathrm{H}, J=6.4 \mathrm{~Hz}), 4.15-4.07(\mathrm{~m}, 4 \mathrm{H}), 3.61(\mathrm{~s}, 4 \mathrm{H}), 3.30(\mathrm{dd}, 1 \mathrm{H}, J=5.5 \mathrm{~Hz}, 9.6 \mathrm{~Hz}), 2.90-2.81$ (m, 1H), 2.19-2.08 (m, 1H), 2.01-1.90 (m, 1H), 1.24-1.13 (m, 6H). ${ }^{13} \mathrm{C}-\mathrm{NMR}\left(100 \mathrm{MHz}, \mathrm{CDCl}_{3}, 25{ }^{\circ} \mathrm{C}\right)$ $\delta /$ ppm: 73.8, 168.5, 168.4, 145.3, 130.0 (quartet, $J=32 \mathrm{~Hz}$ ), 126.7, 125.2 (quartet, $J=4 \mathrm{~Hz}$ ), 123.9 (quartet, $J=270 \mathrm{~Hz}), 74.2,61.5,61.4,52.7,50.2,49.6,27.5,13.6 . \mathrm{MS}(20 \mathrm{eV}, \mathrm{EI}) \mathrm{m} / z(\%): 420$ (3) $\left[\mathrm{M}^{+}, 403\right.$ (14), 360 (69), 245 (34), 173 (27), 160 (100), 126 (42), 55 (20). HRMS (ESI) for $\mathrm{C}_{19} \mathrm{H}_{23} \mathrm{~F}_{3} \mathrm{NaO}_{7},[\mathrm{M}+\mathrm{Na}]^{+}$(443.1294) found: 400.1288 .

Erythro-1,1-diethyl 3-methyl 4-(4-cyanophenyl)-4-hydroxybutane-1,1,3-tricarboxylate (erythro-4e). ${ }^{1} \mathrm{H}-\mathrm{NMR}\left(400 \mathrm{MHz}, \mathrm{CDCl}_{3}, 25{ }^{\circ} \mathrm{C}\right) \delta / \mathrm{ppm}: 7.65(\mathrm{~d}, 2 \mathrm{H}, J=8.4 \mathrm{~Hz}), 7.48(\mathrm{~d}, 2 \mathrm{H}, J=8.2 \mathrm{~Hz}), 5.10$ (pseudo t, 1H, $J=3.9 \mathrm{~Hz}), 4.20-4.07(\mathrm{~m}, 4 \mathrm{H}), 3.66(\mathrm{~s}, 3 \mathrm{H}), 3.36(\mathrm{dd}, 1 \mathrm{H}, J=5.4 \mathrm{~Hz}, 9.6 \mathrm{~Hz}), 3.20$ (d, $J=3.4 \mathrm{~Hz}), 2.88-2.80(\mathrm{~m}, 1 \mathrm{H}), 2.34-2.24(\mathrm{~m}, 1 \mathrm{H}), 2.15-2.05(\mathrm{~m}, 1 \mathrm{H}), 1.28-1.16(\mathrm{~m}, 6 \mathrm{H}) .{ }^{13} \mathrm{C}-\mathrm{NMR}$ $\left(100 \mathrm{MHz}, \mathrm{CDCl}_{3}, 25^{\circ} \mathrm{C}\right) \delta / \mathrm{ppm}: 174.0,168.9,168.7,146.2,132.2,126.7,118.6,111.7,73.0,61.7$, 
61.6, 52.2, 49.8, 49.7, 25.3, 14.0. HRMS (MALDI) for $\mathrm{C}_{19} \mathrm{H}_{23} \mathrm{NNaO}_{7},[\mathrm{M}+\mathrm{Na}]^{+}$(400.1372) found: 400.1385 .

Threo-1,1-diethyl 3-methyl 4-(4-cyanophenyl)-4-hydroxybutane-1,1,3-tricarboxylate (threo-4e). ${ }^{1} \mathrm{H}-\mathrm{NMR}\left(400 \mathrm{MHz}, \mathrm{CDCl}_{3}, 25{ }^{\circ} \mathrm{C}\right) \delta / \mathrm{ppm}: 7.61(\mathrm{~d}, 2 \mathrm{H}, J=8.3 \mathrm{~Hz}), 7.41(\mathrm{~d}, 2 \mathrm{H}, J=8.2 \mathrm{~Hz}), 4.87(\mathrm{~d}$, $1 \mathrm{H}, J=6.2 \mathrm{~Hz}), 4.18-4.07(\mathrm{~m}, 4 \mathrm{H}), 3.60(\mathrm{~s}, 3 \mathrm{H}), 3.55$ (brs, $1 \mathrm{H}), 3.30$ (dd, $1 \mathrm{H}, J=5.6 \mathrm{~Hz}, 9.5 \mathrm{~Hz})$, 2.89-2.80 (m, 1H), 2.20-2.10 (m, 1H), 2.03-1.91 (m, 1H), 1.25-1.16 (m, 6H). ${ }^{13} \mathrm{C}-\mathrm{NMR}(100 \mathrm{MHz}$, $\left.\mathrm{CDCl}_{3}, 25^{\circ} \mathrm{C}\right) \delta / \mathrm{ppm}: 173.7,168.5,168.4,146.7,132.2,127.0,118.5,111.7,74.0,61.7,61.6,52.0$, 49.9, 49.5, 27.7, 13.9. MS (20 eV, EI) $m / z(\%): 78$ (14) [M+1] $]^{+}, 360$ (13), 317 (39), 300 (29), 282 (16), 246 (21), 203 (31), 186 (29), 160 (100), 133 (15). HRMS (MALDI) for $\mathrm{C}_{19} \mathrm{H}_{23} \mathrm{NNaO}_{7},[\mathrm{M}+\mathrm{Na}]^{+}$ (400.1372) found: 400.1385.

1,1-Diethyl 3-methyl 4-hydroxy-4-(pyridin-3-yl)butane-1,1,3-tricarboxylate (4f). ${ }^{1} \mathrm{H}-\mathrm{NMR}$ (400 MHz, $\left.\mathrm{CDCl}_{3}, 25^{\circ} \mathrm{C}\right) \delta / \mathrm{ppm}: 8.44(\mathrm{~s}, 2 \mathrm{H}), 7.71-7.61(\mathrm{~m}, 2 \mathrm{H}), 7.50-7.40(\mathrm{~m}, 1 \mathrm{H}), 7.27-7.22(\mathrm{~m}, 1 \mathrm{H}), 4.99$ and $4.85(\mathrm{~d}, 1 \mathrm{H}, J=5.9 \& 7.2 \mathrm{~Hz}), 4.42(\mathrm{brs}, 1 \mathrm{H}), 4.19-4.04(\mathrm{~m}, 4 \mathrm{H}), 3.65$ and $3.55(\mathrm{~s}, 3 \mathrm{H}), 3.44-3.22$ (m, 1H), 2.95-2.74 (m, 1H), 2.36-1.86 (m, 1H), 1.29-1.12 (m, 6H). ${ }^{13} \mathrm{C}-\mathrm{NMR}\left(100 \mathrm{MHz}, \mathrm{CDCl}_{3}, 25{ }^{\circ} \mathrm{C}\right)$ $\delta /$ ppm: 173.8, 173.5, 168.9, 168.7, 168.6, 168.4, 149.2, 148.1, 137.1, 134.2, 134.1, 131.6, 130.8, 130.7, $128.6,128.5,123.5,72.7,71.6,61.6,61.5,61.4,51.9,51.8,50.4,50.2,49.9,49.7,27.7,26.0,13.9$.

Threo-triethyl 4-hydroxy-4-(4-nitrophenyl)butane-1,1,3-tricarboxylate (threo-4g). ${ }^{1} \mathrm{H}-\mathrm{NMR}$ (400 MHz, $\left.\mathrm{CDCl}_{3}, 25^{\circ} \mathrm{C}\right) \delta / \mathrm{ppm}: 8.16(\mathrm{~d}, 2 \mathrm{H}, J=8.7 \mathrm{~Hz}), 7.48(\mathrm{~d}, 2 \mathrm{H}, J=8.7 \mathrm{~Hz}), 4.93(\mathrm{t}, 1 \mathrm{H}, J=6.2 \mathrm{~Hz}), 4.20-4.09$ $(\mathrm{m}, 4 \mathrm{H}), 4.09-4.01(\mathrm{~m}, 2 \mathrm{H}), 3.68(\mathrm{~d}, 1 \mathrm{H}, J=6.7 \mathrm{~Hz}), 3.33$ (dd, 1H, $J=5.5 \mathrm{~Hz}, 9.6 \mathrm{~Hz}), 2.90-2.81$ (m, $1 \mathrm{H}), 2.24-2.13(\mathrm{~m}, 1 \mathrm{H}), 2.09-2.00(\mathrm{~m}, 1 \mathrm{H}), 1.21(\mathrm{t}, 3 \mathrm{H}, J=7.2), 1.12(\mathrm{t}, 3 \mathrm{H}, J=7.2) .{ }^{13} \mathrm{C}-\mathrm{NMR}$ $\left(100 \mathrm{MHz}, \mathrm{CDCl}_{3}, 25{ }^{\circ} \mathrm{C}\right) \delta / \mathrm{ppm}: 173.3,168.6,168.4,148.8,147.4,127.1,123.5,73.6,61.7,61.6$, 61.2, 49.8, 49.5, 27.9, 13.9, 13.8. MS (MALDI) $m / z(\%): 434$ (100) [M+Na], 368 (55), 352 (63). HRMS (MALDI) for $\mathrm{C}_{19} \mathrm{H}_{25} \mathrm{NNaO}_{9},[\mathrm{M}+\mathrm{Na}]^{+}$(434.1427) found: 434.1436.

Threo-triethyl 4-hydroxy-4-(3-nitrophenyl)butane-1,1,3-tricarboxylate (threo-4h). ${ }^{1} \mathrm{H}-\mathrm{NMR}$ (400 MHz, $\left.\mathrm{CDCl}_{3}, 25^{\circ} \mathrm{C}\right) \delta / \mathrm{ppm}: 8.18,(\mathrm{~s}, 1 \mathrm{H}), 8.15-8.09(\mathrm{~m}, 1 \mathrm{H}), 7.66(\mathrm{~d}, 1 \mathrm{H}, J=7.7 \mathrm{~Hz}), 7.52,(\mathrm{~d}, 1 \mathrm{H}, J=7.9 \mathrm{~Hz})$, 4.96 (t, 1H, $J=5.2 \mathrm{~Hz}$ ), 4.21-4.12 (m, 4H), 4.09 (quartet, $2 \mathrm{H}, J=7.2 \mathrm{~Hz}), 3.60$ (d, 1H, $J=6.2 \mathrm{~Hz}$ ), $3.37(\mathrm{dd}, 1 \mathrm{H}, J=5.5 \mathrm{~Hz}, 9.5 \mathrm{~Hz}), 2.95-2.84(\mathrm{~m}, 1 \mathrm{H}), 2.28-2.18(\mathrm{~m}, 1 \mathrm{H}), 2.14-2.03(\mathrm{~m}, 1 \mathrm{H}), 1.28-1.19$ $(\mathrm{m}, 6 \mathrm{H}), 1.14(\mathrm{t}, 3 \mathrm{H}, J=7.2 \mathrm{~Hz}) \cdot{ }^{13} \mathrm{C}-\mathrm{NMR}\left(100 \mathrm{MHz}, \mathrm{CDCl}_{3}, 25{ }^{\circ} \mathrm{C}\right) \delta / \mathrm{ppm}: 173.3,168.6,168.5$, $148.3,143.7,132.3,129.4,122.9,121.2,73.7,61.7,61.6,61.2,49.8,49.6,27.9,13.9 . \mathrm{MS}$ (70 eV, EI) m/z (\%):412 (4) [M+1] $]^{+}, 393$ (5), 320 (14), 302 (15), 260 (25), 185 (39), 160 (100), 133 (15), 55 (16). HRMS (MALDI) for $\mathrm{C}_{19} \mathrm{H}_{25} \mathrm{NNaO}_{9},[\mathrm{M}+\mathrm{Na}]^{+}$(434.1427) found: 434.1431 .

Threo-triethyl 4-(4-cyanophenyl)-4-hydroxybutane-1,1,3-tricarboxylate (threo-4i). ${ }^{1} \mathrm{H}-\mathrm{NMR}$ (400 MHz, $\left.\mathrm{CDCl}_{3}, 25{ }^{\circ} \mathrm{C}\right) \delta / \mathrm{ppm}: 7.65(\mathrm{~d}, 2 \mathrm{H}, J=8.2 \mathrm{~Hz}), 7.45(\mathrm{~d}, 2 \mathrm{H}, J=8.2 \mathrm{~Hz}), 4.91(\mathrm{t}, 1 \mathrm{H}, J=6.4 \mathrm{~Hz})$, 4.25-4.14 (m, 4H), 4.09 (quartet, $J=7.0 \mathrm{~Hz}), 3.38(\mathrm{dd}, 1 \mathrm{H}, J=5.7 \mathrm{~Hz}, 9.4 \mathrm{~Hz}), 3.34$ (d, 1H, $J=7.2 \mathrm{~Hz})$, 2.93-2.83 (m, 1H), 2.31-2.20 (m, 1H), 2.19-2.07 (m, 1H), $1.26(\mathrm{t}, 6 \mathrm{H}, J=7.2 \mathrm{~Hz}), 1.15$ (t, 3H, $J=7.1 \mathrm{~Hz})$. ${ }^{13} \mathrm{C}-\mathrm{NMR}\left(100 \mathrm{MHz}, \mathrm{CDCl}_{3}, 25{ }^{\circ} \mathrm{C}\right) \delta / \mathrm{ppm}: 173.1,168.5,168.3,146.8,132.0,126.9,118.4,111.5$, 73.9, 61.5, 61.4, 61.0, 49.8, 49.4, 27.7, 13.8. MS (20 eV, EI) $m / z$ (\%): 391 (4) [M] $]^{+}, 218$ (19), 160 (100), 132 (10), 102 (30). HRMS (MALDI) for $\mathrm{C}_{20} \mathrm{H}_{25} \mathrm{NNaO}_{7},[\mathrm{M}+\mathrm{Na}]^{+}$(414.1529) found: 414.1535. 
Threo-ethyl 3-(4-cyanophenyl)-2-((N,4-dimethylphenylsulfonamido)methyl)-3-hydroxypropanoate (threo-4j). ${ }^{1} \mathrm{H}-\mathrm{NMR}\left(400 \mathrm{MHz}, \mathrm{CDCl}_{3}, 25{ }^{\circ} \mathrm{C}\right) \delta / \mathrm{ppm}: 8.35(\mathrm{~s}, 2 \mathrm{H}), 7.65(\mathrm{~d}, 1 \mathrm{H}, J=7.9 \mathrm{~Hz}), 7.22(\mathrm{dd}$, $1 \mathrm{H}, J=4.8 \mathrm{~Hz}, J=7.7 \mathrm{~Hz}), 4.81(\mathrm{~d}, 1 \mathrm{H}, J=7.2 \mathrm{~Hz}), 4.16-3.96(\mathrm{~m}, 6 \mathrm{H}), 3.26(\mathrm{dd}, 1 \mathrm{H} J=6.2 \mathrm{~Hz}, 10.1 \mathrm{~Hz})$, 2.85-2.74 (m, 1H), 2.14-2.01 (m, 1H), 1.94-1.82 (m, 1H), 1.22-1.09 (m, 9H). ${ }^{13} \mathrm{C}-\mathrm{NMR}(100 \mathrm{MHz}$, $\left.\mathrm{CDCl}_{3}, 25^{\circ} \mathrm{C}\right) \delta / \mathrm{ppm}: 173.2,168.5,168.4,148.7,147.8,137.3,134.1,123.5,72.5,61.5,61.4,60.9$, 50.2, 49.6, 27.7, 13.9, 13.8, 13.7. MS (MALDI) $\mathrm{m} / z$ (\%): 368 (100) [M+1] $]^{+}, 350$ (38), 305 (50), 289 (37). HRMS (MALDI) for $\mathrm{C}_{18} \mathrm{H}_{26} \mathrm{NO}_{7},[\mathrm{M}+\mathrm{H}]^{+}$(368.1709) found: 368.1719.

Threo-trimethyl 4-hydroxy-4-(4-nitrophenyl)butane-1,1,3-tricarboxylate (threo-4k). ${ }^{1} \mathrm{H}-\mathrm{NMR}$ (400 MHz, $\left.\mathrm{CDCl}_{3}, 25^{\circ} \mathrm{C}\right) \delta / \mathrm{ppm}: 8.20(\mathrm{~d}, 2 \mathrm{H}, J=8.7 \mathrm{~Hz}), 7.50(\mathrm{~d}, 2 \mathrm{H}, J=8.7 \mathrm{~Hz}), 4.96(\mathrm{~d}, 1 \mathrm{H}, J=6.0 \mathrm{~Hz}), 3.71$ $(\mathrm{s}, 6 \mathrm{H}), 3.65(\mathrm{~s}, 3 \mathrm{H}), 3.41(\mathrm{dd}, 1 \mathrm{H}, J=5.7 \mathrm{~Hz}, 9.3 \mathrm{~Hz}), 2.92-2.88(\mathrm{~m}, 1 \mathrm{H}), 2.24-2.19(\mathrm{~m}, 1 \mathrm{H})$, 2.13-2.08 (m, 1H). ${ }^{13} \mathrm{C}-\mathrm{NMR}\left(100 \mathrm{MHz}, \mathrm{CDCl}_{3}, 25{ }^{\circ} \mathrm{C}\right) \delta / \mathrm{ppm}: 173.6,168.9,168.6,148.5,147.6$, 127.1, 123.7, 73.9, 52.8, 52.7, 52.1, 49.8, 49.2, 27.9. MS (20 eV, EI) $m / z(\%): 369$ (8) [M] $]^{+}, 317$ (39), 300 (29), 282 (16), 246 (21), 152 (31), 145 (29), 131 (100), 86 (46). HRMS (MALDI) for $\mathrm{C}_{16} \mathrm{H}_{19} \mathrm{NNaO}_{9},[\mathrm{M}+\mathrm{Na}]^{+}$(392.0957) found: 369.0968.

Erythro-methyl 2-(hydroxy(4-nitrophenyl)methyl)-4,4-bis(phenylsulfonyl)butanoate (erythro-5). mp.: 96.1-96.5 ${ }^{\circ} \mathrm{C} .{ }^{1} \mathrm{H}-\mathrm{NMR}\left(400 \mathrm{MHz}, \mathrm{CDCl}_{3}, 25{ }^{\circ} \mathrm{C}\right) \delta / \mathrm{ppm}: 8.21(\mathrm{~d}, 2 \mathrm{H}, J=8.6 \mathrm{~Hz}), 7.88(\mathrm{~d}, 2 \mathrm{H}$, $J=7.4 \mathrm{~Hz}$ ), 7.74-7.60 (m, 4H), 7.60-7.49 (m, 4H), 7.45 (t, 2H, $J=7.9), 5.26(\mathrm{t}, 1 \mathrm{H}, J=4.2 \mathrm{~Hz}), 4.87$ $(\mathrm{dd}, 1 \mathrm{H}, J=3.2 \mathrm{~Hz}, 7.9 \mathrm{~Hz}), 3.60(\mathrm{~s}, 3 \mathrm{H}), 3.41-3.31(\mathrm{~m}, 1 \mathrm{H}), 3.12(\mathrm{~d}, J=3.8 \mathrm{~Hz}), 2.72-2.60(\mathrm{~m}, 1 \mathrm{H})$, 2.31-2.19 (m, 1H). ${ }^{13} \mathrm{C}-\mathrm{NMR}\left(100 \mathrm{MHz}, \mathrm{CDCl}_{3}, 25{ }^{\circ} \mathrm{C}\right) \delta / \mathrm{ppm}: 172.7,147.9,147.5,137.4,136.8$, 134.7, 129.6, 129.4, 129.2, 129.0, 127.2, 123.6, 80.6, 72.5, 52.5, 49.7, 22.5. HRMS (ESI) for $\mathrm{C}_{24} \mathrm{H}_{23} \mathrm{NNaO}_{9} \mathrm{~S}_{2},[\mathrm{M}+\mathrm{Na}]^{+}$(556.0712) found: 556.0717. CCDC number: 837000 .

Threo-methyl 2-(hydroxy(4-nitrophenyl)methyl)-4,4-bis(phenylsulfonyl)butanoate (threo-5). mp.: 140.2-140.7 ${ }^{\circ} \mathrm{C} .{ }^{1} \mathrm{H}-\mathrm{NMR}\left(400 \mathrm{MHz}, \mathrm{CDCl}_{3}, 25{ }^{\circ} \mathrm{C}\right) \delta / \mathrm{ppm}: 8.18(\mathrm{~d}, 2 \mathrm{H}, J=8.6 \mathrm{~Hz}), 7.86(\mathrm{~d}, 2 \mathrm{H}$, $J=7.4 \mathrm{~Hz}), 7.81(\mathrm{~d}, 2 \mathrm{H}, J=7.7 \mathrm{~Hz}), 7.69(\mathrm{t}, 2 \mathrm{H}, J=7.5 \mathrm{~Hz}), 7.60-7.49(\mathrm{~m}, 4 \mathrm{H}), 7.47(\mathrm{t}, 2 \mathrm{H}$, $J=8.6), 5.07(\mathrm{t}, 1 \mathrm{H}, J=6.0 \mathrm{~Hz}), 4.73(\mathrm{dd}, 1 \mathrm{H}, J=4.0 \mathrm{~Hz}, 7.6 \mathrm{~Hz}), 3.57(\mathrm{~s}, 3 \mathrm{H}), 3.54-3.45(\mathrm{~m}, 1 \mathrm{H})$, $3.36(\mathrm{~d}, J=6.5 \mathrm{~Hz}), 2.54-2.42(\mathrm{~m}, 1 \mathrm{H}), 2.38-2.26(\mathrm{~m}, 1 \mathrm{H}),{ }^{13} \mathrm{C}-\mathrm{NMR}\left(100 \mathrm{MHz}, \mathrm{CDCl}_{3}, 25{ }^{\circ} \mathrm{C}\right)$ $\delta /$ ppm: 172.4, 147.8, 147.6, 137.2, 137.1, 134.8, 134.7, 129.6, 129.5, 129.2, 129.1, 127.2, 123.7, 80.6, 73.4, 52.4, 49.9, 24.8. HRMS (ESI) for $\mathrm{C}_{24} \mathrm{H}_{23} \mathrm{NNaO}_{9} \mathrm{~S}_{2}$, [M+Na] $]^{+}$(556.0712) found: 556.0710.

2,2-Diethyl 4-methyl 5-hydroxy-5-(4-nitrophenyl)pentane-2,2,4-tricarboxylate (6). ${ }^{1} \mathrm{H}-\mathrm{NMR}(400 \mathrm{MHz}$, $\left.\mathrm{CDCl}_{3}, 25^{\circ} \mathrm{C}\right) \delta / \mathrm{ppm}: 8.18(\mathrm{~d}, 2 \mathrm{H}, J=8.7 \mathrm{~Hz}), 7.47(\mathrm{~d}, 2 \mathrm{H}, J=8.7 \mathrm{~Hz}), 4.99$ (pseudo t, $1 \mathrm{H}, J=5.5 \mathrm{~Hz}$ ), $4.17-4.11(\mathrm{~m}, 4 \mathrm{H}), 3.56(\mathrm{~s}, 4 \mathrm{H}), 2.98-2.97(\mathrm{~m}, 1 \mathrm{H}), 2.31(\mathrm{dd}, 1 \mathrm{H}, J=14.7,9.2 \mathrm{~Hz}), 2.15(\mathrm{dd}, 1 \mathrm{H}$, $J=14.7,2.6 \mathrm{~Hz}), 1.36$ (s, 3H), $1.24-1.17$ (m, 6H). ${ }^{13} \mathrm{C}-\mathrm{NMR}\left(100 \mathrm{MHz}, \mathrm{CDCl}_{3}, 25{ }^{\circ} \mathrm{C}\right) \delta / \mathrm{ppm}: 174.0$, $171.9,171.5,148.8,147.5,127.0,123.5,74.8,61.7,61.6,52.9,51.9,48.6,34.3,20.2,13.9,13.8 . \mathrm{MS}$ (ESI) $m / z(\%): 434(100)[\mathrm{M}+\mathrm{Na}]^{+}, 305$ (5). HRMS (MALDI) for $\mathrm{C}_{19} \mathrm{H}_{25} \mathrm{NNaO}_{9},[\mathrm{M}+\mathrm{Na}]^{+}(434.1427)$ found: 434.1438 . 


\section{Conclusions}

In summary, we have developed a novel, simple approach toward the synthesis of highly functionalized molecules via three-component reactions of substituted aromatic aldehydes, alkyl acrylates and activated alkane catalyzed by ethyl diphenylphosphine. Furthermore, we presented the first organocatalytic addition of carbon-nucleophile to the in-situ generated MBH adducts. This multicomponent reaction has a broad reaction scope with all three components of acrylate, aldehyde and activated alkane. Further mechanistic details and development of their asymmetric threecomponent reactions, are now underway in our laboratory.

\section{Supplementary Materials}

Supplementary materials can be accessed at: http://www.mdpi.com/1420-3049/17/3/2529/s1.

\section{Acknowledgements}

We thank the National Science Council of the Republic of China (NSC Grant No. 99-2113-M-003004-MY2) and National Taiwan Normal University (99T3030-6) for financial support.

\section{References and Notes}

1. Dalko, P.I.; Moisan, L. In the golden age of organocatalysis. Angew. Chem. Int. Ed. 2004, 43, 5138-5175.

2. Dalko, P.I.; Moisan, L. Enantioselective organocatalysis. Angew. Chem. Int. Ed. 2001, 40, 3726-3748.

3. Zhu, J., Bienaymé, H., Eds. Multicomponent Reactions; Wiley-VCH: Weinheim, Germany, 2005.

4. Dömling, A.; Ugi, I. Multicomponent reactions with isocyanides. Angew. Chem. 2000, 39, 3168-3210.

5. Bienaymé, H.; Hulme, C.; Oddon, G.; Schmitt, P. Maximizing synthetic efficiency: Multi-component transformations lead the way. Chem. Eur. J. 2000, 6, 3321-3329.

6. Orru, R.V.A.; de Greef, M. Recent advances in solution-phase multicomponent methodology for the synthesis of heterocyclic compounds. Synthesis 2003, 10, 1471-1799.

7. Hulme, C.; Gore, V. Multi-component Reactions: Emerging chemistry in drug discover 'From Xylocain to Crixivan'. Curr. Med. Chem. 2003, 10, 51-80.

8. Ramón, D.J.; Yus, M. Asymmetric multicomponent reactions (AMCRs): The new frontier. Angew. Chem. Int. Ed. 2005, 44, 1602-1634.

9. Dömling, A. Recent developments in isocyanide based multicomponent reactions in applied chemistry. Chem. Rev. 2006, 106, 17-89.

10. Tietze, L.F., Brasche, G., Gericke, K., Eds. Domino Reactions in Organic Synthesis; Wiley-VCH: Weinheim, Germany, 2006.

11. Posner, G.H. Multicomponent one-pot annulations forming 3 to 6 bonds. Chem. Rev. 1986, 86, 831-844.

12. Nicolaou, K.C.; Edmonds, D.J.; Bulger, P.G. Cascade reactions in total synthesis. Angew. Chem. Int. Ed. 2006, 45, 7134-7186. 
13. Basavaiah, D.; Reddy, B.S.; Badsara, S.S. Recent contributions from the Baylis-Hillman reaction to organic chemistry. Chem. Rev. 2010, 110, 5447-5674.

14. Basavaiah, D.; Rao, K.V.; Reddy, R. The Baylis-Hillman reaction: A novel source of attraction, opportunities, and challenges in synthetic chemistry. Chem. Soc. Rev. 2007, 36, 1581-1588.

15. Davie, E.A.C.; Mennen, S.M.; Xu, Y.; Miller, S.J. Asymmetric catalysis mediated by synthetic peptides. Chem. Rev. 2007, 107, 5759-5812.

16. Masson, G.; Housseman, C.; Zhu, J. The enantioselective Morita-Baylis-Hillman reaction and its aza counterpart. Angew. Chem. Int. Ed. 2007, 46, 4614-4628.

17. Basavaiah, D.; Rao, J.A.; Satyanarayana, T. Recent advances in the Baylis-Hillman reaction and applications. Chem. Rev. 2003, 103, 811-891.

18. Langer, P. New strategies for the development of an asymmetric version of the Baylis-Hillman reaction. Angew. Chem. Int. Ed. 2000, 39, 3049-3052.

19. Ciganek, E. The catalyzed $\alpha$-hydroxyalkylation and $\alpha$-aminoalkylation of activated olefins (The Morita-Baylis-Hillman Reaction). In Organic Reactions, Paquette, L.A., Ed.; Wiley: New York, NY, USA, 1997; Volume 51, pp. 201-350.

20. Singh, V.; Batra, S. Advances in the Baylis-Hillman reaction-assisted synthesis of cyclic frameworks. Tetrahedron 2008, 64, 4511-4574.

21. Wang, W.; Yu, M. One-pot sequential Baylis-Hillman and Michael reactions. Tetrahedron Lett. 2004, 45, 7141-7143.

22. Kim, S.C.; Lee, K.Y.; Lee, H.S.; Kim, J.N. Synthesis of poly-substituted benzenes starting from Baylis-Hillman adducts: DBU-assisted unusual dehydrogenation. Tetrahedron 2008, 64, 103-109.

23. Roy, A.K.; Pathak, R.; Yadav, G.P.; Maulik, P.R.; Batra, S. Neighboring-group effect: DBU-promoted ring transformation of substituted isoxazoles to substituted pyrroles. Synthesis 2006, 1021-1027.

24. Nag, S.; Yadav, G.P.; Maulik, P.R.; Batra, S. Sodium hydride mediated cascade reaction towards the synthesis of 1,5-disubstituted uracil from cyanamides derived from Baylis-Hillman adducts. Synthesis 2007, 911-917.

25. Wang, D.-W.; Syu, S.; Hung, Y.-T.; Chen, P.; Lee, C.-J.; Chen, K.-W.; Chen Y.-J.; Lin, W. Organocatalytic tandem three-component reaction of aldehyde, alkyl vinyl ketone, and amide: One-pot syntheses of highly functional alkenes. Org. Biomol. Chem. 2011, 9, 363-366.

26. Syu, S.; Wang, D.-W.; Chen, P.; Hung, Y.-T.; Jhang, Y.-W.; Kao, T.-T.; Lee Y.-T.; Lin, W. Tandem three-component reaction of aldehyde, alkyl acrylate, and amide using ethyl diphenylphosphine as organocatalyst. Tetrahedron Lett. 2010, 51, 5943-5946.

27. Syu, S.; Lee Y.-T.; Jang, Y.-J.; Lin, W. Organocatalytic tandem three-component reaction of imine, alkyl vinyl ketone, and imide via aza-Baylis-Hillman reaction. J. Org. Chem. 2011, 76, 2888-2891.

28. The proposed mechanism concerning the phosphine catalysis process was not mentioned in this report. For detailed information, please see the references [25-27].

29. Methot, J.L.; Roush, W.R. Nucleophilic phosphine organocatalysis. Adv. Synth. Cat. 2004, 346, $1035-1050$. 
30. Wang, L.-C.; Luis, A.L.; Agapiou, K.; Jang, H.-Y.; Krische, M.J. Organocatalytic michael cycloisomerization of bis (enones): The intramolecular Rauhut-Currier reaction. J. Am. Chem. Soc. 2002, 124, 2402-2403.

31. Lu, C.; Lu, X. Tandem reactions to construct heterocycles via phosphine-catalyzed umpolung addition and intramolecular conjugate addition. Org. Lett. 2002, 4, 4677-4679.

32. Xu, L.-W.; Xia, C.-G. Highly efficient phosphine-catalyzed aza-Michael reactions of $\alpha, \beta$-unsaturated compounds with carbamates in the presence of TMSCl. Tetrahedron Lett. 2004, 45, 4507-4510.

33. Carolina, G.; Maria, L.; Caroline, M.; Marcial, M.-M.; Rosa, M.S.; Adelina, V. Michael additions catalyzed by phosphines. An overlooked synthetic method. Tetrahedron 2005, 61, 8598-8605.

34. Yuan, K.; Song, H.-L.; Hu, Y.; Wu, X.-Y. Chiral phosphinothiourea-catalyzed asymmetric Morita-Baylis-Hillman reactions of acrylates with aromatic aldehydes. Tetrahedron 2009, 65, 8185-8190.

35. The relative configuration of $\mathbf{5}$ was confirmed by X-ray analysis (CCDC number: 837000 for erythro-5). The stereochemistry of $\mathbf{4}$ and $\mathbf{6}$ was determined by ${ }^{1} \mathrm{H}$ NMR analysis in comparison to $\mathbf{5}$.

Sample Availability: Samples of all compounds are available from the authors.

(C) 2012 by the authors; licensee MDPI, Basel, Switzerland. This article is an open access article distributed under the terms and conditions of the Creative Commons Attribution license (http://creativecommons.org/licenses/by/3.0/). 